\title{
Parâmetros metabólicos em vacas leiteiras durante o período de transição pós-parto
}

\section{Metabolic profiles during the pospartum transition period in dairy cows}

\author{
Ernani Paulino Lago, ${ }^{\star}$ Ana Paula Delgado da Costa, ${ }^{* *}$ Alexandre Vaz Pires, ${ }^{* *}$ Ivanete Susin, ${ }^{* \star *}$ Vidal Pedroso de Farias ${ }^{\star \star *}$ \\ Luiz Alberto do Lago***
}

\section{Resumo}

A determinação dos parâmetros metabólicos considerados normais em vacas leiteiras de alta produção é fundamental para a avaliação do metabolismo de vacas no período de transição. No presente trabalho, foram determinadas as concentrações sangüíneas de glicose, ácidos graxos não-esterificados (AGNE), beta-hidroxibutirato (BHBA) e aspartato aminotransferase (AST) de 70 vacas holandesas consideradas clinicamente sadias para as principais doenças puerperais durante as primeiras oito semanas de lactação, com o objetivo de se determinar os parâmetros metabólicos normais sob condições observadas aqui no Brasil. Os valores médios encontrados foram 45,54 $( \pm 7,17) \mathrm{mg} / \mathrm{dL}, 4,04( \pm 1,45) \mu \mathrm{mol} / \mathrm{L}, 2,97( \pm 1,04) \mathrm{mg} / \mathrm{dL}$ e 79,44 $( \pm 23,25) \mathrm{UI} / \mathrm{L}$, para glicose, AGNE, BHBA e AST, respectivamente. Analisando cada semana separadamente, foi observado que as concentrações de glicose apresentaram-se aumentadas na primeira semana, permanecendo constantes nas demais semanas. As concentrações de AGNE apresentaram-se aumentadas na primeira semana, sendo notada uma diminuição nas demais semanas, o mesmo comportamento observado nas concentrações de BHBA. Já as concentrações de AST permaneceram constantes em todas as semanas. A determinação dos valores normais dos parâmetros metabólicos de vacas e suas variações durante todo o ciclo de produção leiteira é essencial para o entendimento do metabolismo destes animais.

Palavras-chave: vacas leiteiras, período de transição, parâmetros metabólicos.

\begin{abstract}
The determination of metabolic profiles in high-producing dairy cows is essential to assess the metabolism in the transition period. In this work, seventy dairy cows without puerperal diseases were used in order to determine the normal metabolic profiles during the first eight weeks of lactation. The metabolic profiles measured in blood sample were glucose, non-esterified fatty acid (NEFA), B- hydroxybutyrate (BHB) and aspartate-aminotransferase (AST). The mean values observed during the first eight weeks were 45,54 $( \pm 7,17) \mathrm{mg} / \mathrm{dL}, 4,04( \pm 1,45) \mu \mathrm{mol} / \mathrm{L}, 2,97( \pm 1,04) \mathrm{mg} / \mathrm{dL}$ and $79,44( \pm 23,25) \mathrm{UI} / \mathrm{L}$, for glucose, NEFA, BHB and AST, respectively. These determinations were analyzed weekly too. Glucose concentrations were increased at first week but reduced and became constant in the following weeks. For NEFA and BHB, an increase in the first week followed by a decrease in next weeks was observed. AST concentrations showed no variation during all the experiment. The determination of normal metabolic profiles during milk production cycle is essential to understand the dairy cattle metabolism.
\end{abstract}

Keywords: dairy cows, transition period, metabolic profiles.

\section{Introdução}

Em vários países tem havido grande interesse no estudo do metabolismo de vacas leiteiras de alta produção, principalmente durante o período de transição. O período de transição é constituído por duas fases, sendo a primeira formada pelas três últimas semanas que antecedem o parto e a segunda pelas três primeiras semanas pós-parto (Grummer, 1995). Este período é marcado por mudanças endócrinas e metabólicas que preparam as vacas para o parto e a lactação. $O$ fim da fase não-lactante e o início da lactante, juntamente com o estresse do parto, acarretam mudanças que afetam $o$ consumo de alimentos (Vasque-Anon et al., 1994) e o metabolismo das vacas leiteiras (Bell, 1995; Grummer, 1995).

Herdt (1988) observou que o fim da gestação e o início da lactação representam uma difícil fase para a vaca leiteira, devido à grande demanda metabólica. Além do grande requerimento de energia, há também necessidade de enormes quantidades de glicose para sustentar a síntese de lactose pela glândula mamária. Vacas de alta produção podem requerer até $80 \%$ do suprimento total de glicose para produzirem leite. Nesta fase, é comum o aparecimento de transtornos metabólicos que afetam a saúde e a produção

\footnotetext{
"Docente do Departamento de Veterinária - Universidade Federal de Viçosa - Viçosa/MG - e-mail: eplago@ufv.mail.br

" Pós-graduanda do Departamento de Veterinária - Universidade Federal de Viçosa - Viçosa/MG

*** Docente do Departamento de Zootecnia - Escola Superior de Agricultura Luiz de Queirós - ESALQ- USP- Piracicaba/SP

${ }^{*}$ Docente do Departamento de Veterinária - Universidade Federal de Minas Gerais - Belo Horizonte/MG
} 
das vacas leiteiras de alta produção, sendo necessário monitoramento clínico e laboratorial.

Uma forma de se avaliar o metabolismo de vacas no pósparto é por meio da determinação dos parâmetros metabólicos que auxiliam no diagnóstico das principais doenças puerperais (Gonzalez, 1997), tais como o deslocamento de abomaso (Geishauser et al., 1997), a cetose (Drackley et al., 1992) e a lipidose hepática (Gerloff, 1986; Roberts e Reid, 1986; Grummer, 1993). Segundo Whitaker (2000), a análise dos metabólitos sangüíneos em vacas leiteiras, juntamente com dados de produção, pico e curva de lactação e variações no escore de condição corporal é útil para avaliar o perfil reprodutivo e nutricional do animal, bem como auxiliar na prevenção e no diagnóstico de distúrbios metabólicos. Embora diferenças genéticas dos rebanhos, ambiente e manejo possam interferir no metabolismo, os parâmetros metabólicos mais utilizados são glicose, ácidos graxos não-esterificados (AGNE), betahidroxibutirato (BHBA) (Andersson, 1988) e aspartato aminotransferase (AST) (Gerloff, 1986; Zurek et al., 1994).

O principal regulador do metabolismo de energia, nos mamíferos, é a glicose sangüínea (Lehninger et al., 1995). Nos ruminantes, esta possui maior importância, pois apenas 5\% da glicose ingerida é absorvida diretamente (Herdt, 1988). Grande parte da glicose oriunda da dieta sofre fermentação rumenal, sendo convertida a ácidos graxos voláteis e, com isto, faz-se necessário um constante estado de gliconeogênese para suprir a demanda de glicose (Herdt, 1988).

Nas primeiras semanas de lactação, as vacas leiteiras de alta produção apresentam balanço energético negativo, pois a alta demanda energética não é suprida pelo consumo de alimentos, há ineficiência de consumo (Vasque-Anon et al., 1994). O pico de consumo de matéria seca não ocorre antes de oito a dez semanas pós-parto. Entretanto, o pico de lactação ocorre entre a $4^{a}$ e $6^{a}$ semanas. Em resposta ao balanço energético negativo e às baixas concentrações séricas de glicose e insulina, há a mobilização do tecido adiposo, com conseqüente aumento nas concentrações de AGNE e BHBA (Radostists et al., 2000).

Segundo Drackley et al. (1992), as concentrações plasmáticas de AGNE e BHBA aumentam durante a cetonemia. Isto ocorre quando a demanda energética da vaca é maior do que a ingestão de nutrientes, havendo maior taxa de lipólise comparada a de lipogênese, resultando em maior hidrólise de triglicerídeos e liberação de AGNE e glicerol. Os AGNE são utilizados como fonte energética nos tecidos, podendo ainda ser redirecionados para o fígado, onde serão completa ou parcialmente oxidados, produzindo $\mathrm{CO}_{2}$ e acetil-CoA, respectivamente. Quando estes são parcialmente oxidados, o acetilCoA originado pode ser convertido a corpos cetônicos, BHBA, acetoacetato e acetona (Lehninger et al., 1995).

A grande demanda energética que ocorre no início da lactação acarreta intensa mobilização das reservas corporais, principalmente do tecido adiposo (Miller et al., 1991; Vasque-Anon et al., 1994). A mobilização de lipídeos resulta em altas concentrações de AGNE na corrente sangüínea (Vasque-Anon et al., 1994), sendo estas utilizadas como um indicador do metabolismo de energia (Gerloff et al., 1986, Grummer, 1993).
Os corpos cetônicos estão normalmente presentes no sangue de ruminantes adultos e constituem importante fonte de energia para estes animais. Suas concentrações são resultados do balanço entre produção no fígado e utilização pelos tecidos periféricos (Foster, 1988). O BHBA é o corpo cetônico mais utilizado para a avaliação do perfil metabólico e detecção de cetose (Andersson, 1988). A determinação dos teores de corpos cetônicos sofre variações induzidas pela alimentação. Vários fatores influenciam a prevalência de hipercetonemia incluindo idade, raça (Andersson, 1988), número de lactações (Radostits et al., 2000) e estação do ano (Tveit et al., 1992). O aumento no aporte de triglicerídeos hepáticos no início do período pós-parto acarreta um aumento da concentração plasmática de BHBA (Grummer, 1995).

Um bom indicador do aumento da atividade gliconeogênica é a dosagem dos teores séricos de AST (Zurek et al., 1994). Teores altos de AST e AGNE associados a teores baixos de glicose são úteis para a avaliação do grau de infiltração de gordura no fígado (Roberts e Reid, 1986).

Segundo Gerloff (1986), em casos avançados de lipidose hepática, os teores de AST estão geralmente aumentados. A relação entre altas concentrações de triglicerídeos hepáticos e teores aumentados de AST ocorre quando há severo comprometimento dos hepatócitos. Geishauser et al. (1997) associaram o aumento das concentrações de AST e BHBA durante as $1^{\mathrm{a}}$ e $2^{\mathrm{a}}$ semanas de lactação à incidência de deslocamento de abomaso à esquerda (DAE), sendo estas determinações úteis para o diagnóstico e prevenção.

Como pode ser observado, nos últimos anos, o estudo do metabolismo de vacas leiteiras no periparto tem auxiliado o entendimento, prevenção e tratamento das principais doenças puerperais. Apesar da importância do entendimento do metabolismo de vacas leiteiras nesta fase, poucos são os estudos realizados no Brasil, sendo utilizados dados de pesquisas realizadas em outros países. Acredita-se que variações genéticas, ambiente e manejo, provavelmente, interfiram nos teores plasmáticos dos metabólitos supracitados, sendo necessária a determinação destes valores sob as condições observadas no Brasil. O presente trabalho teve como objetivo verificar as concentrações plasmáticas de glicose, AGNE, BHBA e AST em vacas leiteiras consideradas clinicamente sadias.

\section{Material e métodos}

Cento e dezoito vacas holandesas, entre a $1^{\text {a }}$ e $8^{a}$ lactação, pertencentes a um rebanho comercial situado no estado de São Paulo e criadas em regime free-stall foram acompanhadas nas oito primeiras semanas de lactação. As vacas foram ordenhadas três vezes ao dia (4, 12 e 20 horas) e receberam alimentação completa com três refeições diárias (4, 10 e 16 horas).

Durante o experimento, foram determinados, semanalmente, a produção de leite com pesagem individualizada, o escore de condição corporal (ECC) (Wildman et al., 1982; Edmolson et al., 1989) e as concentrações plasmáticas de glicose, BHBA, AGNE e AST. O peso vivo foi determinado logo após o parto e na oitava semana de lactação para verificação da perda de peso. 
Os dados de produção leiteira, ECC e peso corporal foram utilizados para a determinação do desempenho das vacas durante o período experimental, auxiliando na avaliação dos parâmetros metabólicos mensurados.

Amostras da ração completa foram colhidas semanalmente e armazenadas $\mathrm{a}-20^{\circ} \mathrm{C}$ para posterior análise bromatológica. Durante as análises foram avaliadas matéria seca, proteína bruta, extrato etéreo, extrativo não nitrogenado e cinzas segundo a AOAC (1990). As determinações de fibra detergente ácido e fibra detergente neutro foram obtidas pelo método de Van Soest et al. (1991).

As vacas foram submetidas a exame clínico durante todo o período experimental e as que apresentaram quaisquer distúrbios à saúde, tais como mastite, cetose, deslocamento de abomaso, metrite e laminite, foram eliminadas do experimento. Apenas as consideradas clinicamente sadias permaneceram no experimento.

Os animais que obtiveram teores séricos de BHBA maiores do que $7,0 \mathrm{mg} / \mathrm{dL}$ também foram retirados do experimento, não possibilitando, com isso, que vacas portadoras de cetose subclínica fossem consideradas sadias.

A colheita semanal de amostras de sangue foi feita a vácuo, em recipientes contendo fluoreto de sódio para a dosagem de glicose e heparina para as demais determinações. As amostras foram colhidas sempre no mesmo horário, entre 9 e 10 horas da manhã, na tentativa de minimizar o efeito das variações diurnas que ocorrem nas concentrações plasmáticas destas substâncias. Durante a colheita, as amostras foram mantidas refrigeradas em isopor contendo gelo até o término do processo. Em seguida, estas amostras foram centrifugadas a $1100 \times \mathrm{g}$. O plasma obtido foi dividido em alíquotas, distribuído em tubos criogênicos de 1,5 ml e armazenado a $-20^{\circ} \mathrm{C}$ para posterior análise.

Os teores plasmáticos de BHBA foram determinados por metodologia enzimática (Willianson,1962) utilizando-se o kit n³10-A (Sigma Diagnostics, USA). A glicose foi quantificada utilizando-se o kit n 315-100 (Sigma Diagnostics, USA), que segue o método da glicose oxidase descrito por Tietz (1982), adaptado para ser utilizado em placas de microtítulo e posterior leitura em aparelho do tipo "Elisa Reader". Os teores plasmáticos de AGNE foram obtidos utilizando o kit NEFA-C (Wako Chemicals), que segue o método enzimático descrito por Johnson e Peters (1993). A enzima hepática aspartato aminotransferase foi determinada utilizando-se o kit AST Reagent-IFCC (Ciba Corning Diagnostics Corp., USA), que se baseia na metodologia da International Federation of Clinical Chemistry (1975).

No final do experimento, 70 vacas holandesas consideradas sadias foram utilizadas para a determinação das concentrações consideradas ideais de BHBA, AGNE, AST e glicose no sangue.

Os dados obtidos foram analisados utilizando-se o programa computacional STATISTICA, v. 5.0. Procedeu-se à análise de variância (ANOVA) em todas as características e, quando necessário, as médias semanais foram avaliadas por meio do teste Tukey $(p \leq 0,05)$ para demonstrar as diferenças entre as semanas.

\section{Resultados e discussão}

A produção leiteira média durante o período experimental foi de $24,43 \pm 7,90 \mathrm{~kg} /$ dia. O escore de condição corporal observado nas vacas no início do experimento foi de 3,5 , sendo observada uma média de $3,01 \pm 0,48$ durante todo período experimental. O peso médio inicial das vacas foi de 586,43 $\mathrm{kg}$ e o final foi de $546,79 \mathrm{~kg}$, havendo perda de $39,64 \mathrm{~kg}$ durante o período experimental.

Os ingredientes utilizados na formulação da ração e sua porcentagem em relação à matéria seca estão discriminados na Tabela 1.

Tabela 1: Ingredientes utilizados na formulação da ração completa oferecida às vacas durante o período experimental

\begin{tabular}{lc}
\hline \multicolumn{1}{c}{ Ingredientes } & ${\text { \% de } \mathrm{MS}^{1}}^{\top}$ \\
\hline Silagem de milho $^{2}$ & 44,70 \\
Milho moído & 28,28 \\
Soja em grão & 7,62 \\
Farelo de soja $^{3}$ & 9,52 \\
Caroço de algodão $_{\text {Minerais e vitaminas }}{ }^{4}$ & 7,62 \\
\hline
\end{tabular}

${ }^{1}$ Matéria seca; ${ }^{2}$ Silagem de milho contendo uréia; ${ }^{3}$ Farelo de soja com $45 \%$ de proteína bruta; ${ }^{4}$ Fósforo $(5,5 \%)$, cálcio $(22,0 \%)$, magnésio $(3,5 \%)$, enxofre $(2,2 \%)$, sódio $(7,0 \%)$, cloreto $(10,8 \%)$, manganês $(1250$ $\mathrm{ppm})$, ferro (500 ppm), cobre (450 ppm), zinco (1550 ppm), selênio (20 ppm), vit. A (90000 Ul/Kg), vit. D (75000 Ul/Kg), vit. E (1000 Ul/Kg).

A composição da dieta está representada na Tabela 2.

Tabela 2: Composição química da dieta determinada após análise bromatológica da ração completa utilizada na alimentação das vacas durante o período experimental

\begin{tabular}{lc}
\hline Análise realizada & $\%$ da MS \\
\hline Matéria seca & 48,00 \\
Proteína bruta & 16,00 \\
Fibra detergente neutro & 38,89 \\
Fibra detergente ácido & 23,47 \\
Extrato etéreo & 4,50 \\
Matéria mineral & 5,50 \\
Extrativo não-nitrogenado & 53,00 \\
Nutrientes digestíveis totais & \\
\hline
\end{tabular}


Tabela 3: Comparação das concentrações plasmáticas de glicose, beta-hidroxibutirato (BHBA), ácidos graxos nãoesterificados (AGNE) e aspartato-aminotransferase (AST) determinadas nas oito primeiras semanas do período pós-parto de 70 vacas leiteiras consideradas sadias

\begin{tabular}{|c|c|c|c|c|c|c|c|c|c|}
\hline \multirow{2}{*}{ Item } & \multicolumn{8}{|c|}{ Semanas } & \multirow{2}{*}{$\begin{array}{c}\text { Média das } \\
8 \text { semanas } \\
(\mu \pm \sigma)\end{array}$} \\
\hline & 1 & 2 & 3 & 4 & 5 & 6 & 7 & 8 & \\
\hline $\begin{array}{c}\text { GLICOSE } \\
(\mathrm{mg} / \mathrm{dL})\end{array}$ & $52,85 a$ & $45,11 b$ & $43,13 b$ & $44,19 b$ & $44,06 b$ & $44,97 b$ & $44,82 b$ & $45,23 b$ & $45,54 \pm 7,17$ \\
\hline $\begin{array}{c}\text { BHBA } \\
(\mathrm{mg} / \mathrm{dL})\end{array}$ & $3,86 a$ & $3,14 b$ & $3,13 b$ & $2,88 b c$ & $2,61 \mathrm{c}$ & $2,76 b c$ & $2,69 b c$ & $2,97 b c$ & $2,97 \pm 1,04$ \\
\hline $\begin{array}{c}\text { AGNE } \\
(\mu \mathrm{mol} / \mathrm{L})\end{array}$ & $5,25 a$ & $4,70 a b$ & $3,93 \mathrm{~cd}$ & $3,93 \mathrm{~cd}$ & $4,12 b c$ & $3,63 \mathrm{~cd}$ & $3,52 \mathrm{c}$ & $3,28 d$ & $4,04 \pm 1,45$ \\
\hline $\begin{array}{l}\text { AST } \\
(\mathrm{UI} / \mathrm{L})\end{array}$ & $75,53 a$ & $76,81 a$ & $75,77 a$ & $77,36 a$ & $75,06 a$ & $78,36 a$ & $78,54 a$ & $75,58 a$ & $76,63 \pm 24,25$ \\
\hline
\end{tabular}

As médias seguidas pela mesma letra, na mesma linha, não diferem entre si pelo Teste de Tukey $(p<0,05)$.

No presente trabalho, a concentração de glicose plasmática apresentou-se maior na $1^{\text {a }}$ semana de lactação $(P<0,05)$, havendo uma diminuição desta na $2^{\mathrm{a}}$ semana. A partir da $2^{\mathrm{a}}$ semana, as concentrações plasmáticas de glicose permaneceram constantes não havendo diferença significativa $(P>0,05)$ entre as médias das semanas 2 a 8 (Tabela 3$)$. A maior concentração observada na $1^{\text {a }}$ semana pode estar relacionada com a proximidade com o parto, como já descrito por Kunz et al. (1985) e Vasque-Anon et al. (1994). Segundo estes autores, as concentrações plasmáticas de glicose geralmente permanecem estáveis, aumentando rapidamente durante o período de transição pré-parto e tendo o seu pico ao parto, época na qual há um aumento drástico. Após o parto há uma diminuição imediata.

Segundo Grummer (1995), o estresse do parto promove depleção na reserva de glicogênio hepático, acarretando aumento das concentrações sanguíneas de glicose.

Radostits et al. (2000) consideram que a concentração plasmática normal de glicose é de $50 \mathrm{mg} / \mathrm{dL}$, sendo valores abaixo destes considerados patológicos. Valores similares são citados por Erickson et al. (1992) 55,4 mg/dL. Kaneko (1997) cita valores entre 45 e 75 mg/dL. Kunz et al. (1985) determinaram que os valores normais em condições nutricionais similares às do nosso experimento são de 45,04 $\mathrm{mg} / \mathrm{dL}$. Estes valores são similares aos observados no presente trabalho $(38,37-52,71 \mathrm{mg} / \mathrm{dL})$. Stober (1993) também determinou valores similares (33- $44 \mathrm{mg} / \mathrm{dL}$ ).

Miller et al. (1991) observaram que as concentrações plasmáticas de glicose foram $7 \%$ maiores no meio da lactação, quando comparados ao início. Drackley et al. (1992) verificaram que as concentrações plasmáticas de glicose variaram pouco até a $4^{\text {a }}$ semana, havendo aumento a partir da $5^{\text {a }}$ semana, com pico na $8^{a}$ semana. Segundo VasqueAnon et al. (1994) e Putman et al. (1999), o pico da glicose ocorre ao parto como também foi observado por Grummer (1995), sendo este seguido por queda ainda na $1^{\text {a }}$ semana. Após esta fase há aumento progressivo das concentrações de glicose até a $4^{\mathrm{a}}$ semana.
As concentrações sangüíneas de glicose e AGNE obtidas neste estudo correspondem às observadas por outros pesquisadores.

Com relação ao BHBA, os resultados obtidos demonstram teores mais altos na $1^{\text {a }}$ semana, sendo que estes valores diminuem no decorrer da lactação. Os mesmos resultados foram observados por Drackley et al. (1992).

Estes resultados sugerem que mesmo os animais sadios apresentam balanço energético negativo no início da lactação, como também foi observado por Vasque-Anon et al. (1994). Santos (1996) sugeriu que o aumento do BHBA no início da lactação está relacionado com o aumento da demanda energética para a síntese de leite, sem haver proporcional aumento do consumo de alimentos.

Segundo Nielen et al. (1994), Santos (1996) e Whitaker (2000), concentrações plasmáticas de BHBA acima de $10 \mathrm{mg} / \mathrm{dL}$ são consideradas indicativas de cetose. Entretanto, este valor ainda é controverso. Erickson et al. (1992) sugeriram que os teores plasmáticos normais de BHBA são de $6,50 \mathrm{mg} / \mathrm{dL}$, enquanto Kaneko (1997) cita valores entre 5,9 e 13,9 mg/dL.

Duffield (1995) observou teores mais altos de BHBA nas $1^{\text {a }} \mathrm{e}$ $2^{a}$ semanas de lactação. Bertics et al. (1992) e Vasque-Anon et al. (1994) observaram um pico de BHBA plasmático no primeiro mês pós-parto, sendo sua maior incidência na $2^{\mathrm{a}}$ ou $3^{a}$ semana de lactação. Numa pesquisa realizada por Rukkwamsuk et al. (1999), o pico de BHBA ocorreu na $2^{\mathrm{a}}$ semana, havendo queda progressiva no decorrer das quatro semanas subseqüentes de lactação. $\mathrm{Na} 7^{\text {a }}$ semana as concentrações de BHBA voltaram a aumentar, havendo outro pico na $8^{a}$ semana.

Radostits et al. (2000) associaram as concentrações plasmáticas de BHBA ao consumo de energia. Segundo estes pesquisadores, concentrações de BHBA menores do que $8,33 \mathrm{mg} / \mathrm{dL}$ caracterizam consumo adequado de energia, enquanto as de 8,33 a $16,66 \mathrm{mg} / \mathrm{dL}$ determinam consumo inadequado e as acima de $16,66 \mathrm{mg} / \mathrm{dL}$ indicam subnutrição severa. As concentrações de BHBA observadas no presente trabalho $(1,93$ a $4,01 \mathrm{mg} / \mathrm{dL})$ foram bem abaixo das citadas por outros pesquisadores (Erickson et al., 1992; Nielen et al., 1994; Santos, 1996; Radostits et al., 2000). Este achado é de 
grande importância, tendo em vista que o BHBA é um dos principais metabólitos utilizados na determinação de cetose. Logo, faz-se necessária uma avaliação mais precisa do limiar entre animais sadios e portadores de cetose. Novas pesquisas devem ser feitas no Brasil para se definir com maior precisão os valores normais de BHBA. Como pode ser observado na Tabela 3 , houve diferença significativa $(P<0,05)$ entre as concentrações de BHBA nas oito primeiras semanas de lactação.

A análise de AGNE revelou resultados similares aos de BHBA, com concentrações maiores de AGNE nas $1^{\text {a }}$ e $2^{a}$ semanas $(P<0,05)$, sendo este maior na $1^{\text {a }}$ e diminuindo no decorrer das oito semanas. Houve pequeno aumento das concentrações de AGNE na $5^{\text {a }}$ semana. Foi observada diferença significativa $(P<0,05)$ entre os teores de AGNE nas oito semanas como pode ser observado na Tabela 3.

Rukkwamsuk et al. (1999) e Drackley et al. (1992) observaram, em seus experimentos, que as vacas apresentaram concentrações mais altas de AGNE na $1^{\text {a }}$ semana, sendo que estes valores diminuíram no decorrer da lactação, estando estes menores na $8^{a}$ semana na análise de Rukkwamsuk et al. 1999 e um pouco aumentados na de Drackley et al. (1992).

Segundo Grummer (1995), o rápido aumento do AGNE ao parto, provavelmente, está relacionado com o estresse da vaca na ocasião do parto. As concentrações de AGNE diminuem rapidamente após o parto, mas estas ainda permanecem maiores do que no período pré-parto.

Os resultados obtidos no presente trabalho variam de 2,59 a $5,49 \mu \mathrm{mol} / \mathrm{L}$. Segundo Vasque-Anon et al. (1994), estes valores variam de 3,01 a 6,67 $\mu \mathrm{mol} / \mathrm{L}$. Miller et al. (1991) observaram concentrações plasmáticas de AGNE de 2,24 $\mu \mathrm{mol} / \mathrm{L}$. Whitake (2000) considera normais concentrações abaixo de $7,00 \mu \mathrm{mol} / \mathrm{L}$. Os valores obtidos neste experimento correspondem aos observados por outros autores.

\section{Referências}

ANDERSSON, L. Subclinical ketosis in dairy cows. Veterinary Clinics of North America. Food Animal Practice, v. 4, p. 233-251, 1988.

ASSOCIATION OF OFFICIAL ANALYTICAL CHEMISTS. OfficialMethods of Analysis. 15. ed., Washington, D. C., 1990.

BELL, A. W. Regulation of organic nutrient metabolism during transition from late pregnancy to early lactation. Journal of Animal Science, v. 73, p. 2804-2819, 1995.

BERTICS, S. J.; GRUMMER, R. R.; CADORNIGA-VALINO, C.; STODDARD, E. E. Effect of prepartum dry matter intake on liver triglyceride concentration and early lactation. Journal of Animal Science, v. 75, p. 1914, 1992.

DRACKLEY, J. K.; RICHARD, M. J.;BEITZ, D. C.; YOUNG, J. M. Metabolic changes in dairy cows with ketonemia in response to feed restrition and dietary 1,3-Butanediol. Journal of Dairy Science, v. 75, p. 16221634, 1992.

DUFFIELD, T. F.; KELTON, D. F.; LESLIE, K. E. A survey of subclinical ketosis in Ontario dairy cattle. Dairy Research Report, n. 395, p. 2427, 1995.

EDMOLSON, A. J.; LEAN, I. J.; WEAVER, L. D.; FARVER, T.; WEBSTER, G. A body condition scoring chat for Holstein dairy cows. Journal of Dairy Science, v. 72, p. 68, 1989.

ERICKSON, P. S.; MURPHY, M.R.; CLARK, J. H. Supplementation of dairy cow diets with calcium salts of long-chain acids and nicotinic acid in early lactation. Journal of Dairy Science, v. 75, p. 1078-1089, 1992.
Já as concentrações de AST permaneceram constantes durante as oito semanas (Tabela 3 ). Este achado determina que não houve aumento patológico da atividade gliconeogênica, pois, como supracitado, teores altos de AST e AGNE associados a baixas concentrações de glicose auxiliam na determinação de infiltração de gordura no fígado (Roberts e Reid, 1986).

Gregory et al. (1999), em um experimento realizado aqui no Brasil, determinaram que os valores normais de AST em vacas Jersey são de 33,91 ( $\pm 10,99)$ UI/L. Stober et al. (1993) citam valores de 10 a $50 \mathrm{UI} / \mathrm{L}$. Kaneko (1997) cita valores entre 78 e 132 UI/L como sendo normais para bovinos. Entretanto, estes valores segundo Gerloff (1986) são considerados indicativos de lipidose hepática. Segundo este pesquisador, valores acima de $100 \mathrm{UI} / \mathrm{L}$ caracterizam a existência de lipidose. No presente trabalho, foram observados valores entre 53,19 e $99,69 \mathrm{UI} / \mathrm{L}$.

\section{Conclusão}

Os resultados obtidos na análise das concentrações sangüíneas de BHBA e AGNE sugerem que mesmo os animais sadios apresentam um balanço energético negativo no início da lactação.

Novas pesquisas devem ser feitas no Brasil para se definir com maior precisão os valores normais de BHBA.

Em relação aos teores de AST, estes permaneceram constantes durante todo o período experimental, caracterizando que não houve aumento patogênico da atividade gliconeogênica nesta fase.

A determinação dos valores normais dos parâmetros metabólicos de vacas e suas variações durante todo o ciclo de produção leiteira é essencial para o entendimento do metabolismo destes animais.

FOSTER, L. A. Clinical Ketosis. Veterinary Clinics of North America. Food Animal Practice, v. 4, p. 253-267, 1988.

GEISHAISER, T.; LESLIE, K.; DUFFIELD, T.; EDGE, V. An evaluation of milk ketone tests for the prediction of left displace abomasum in dairy cows. Journal of Dairy Science, v. 80, p. 3188-3192, 1997.

GERLOFF, B. J.; HERDT, T. H.; EMERY, R. S. Relationship of hepatic lipidosis to health and performance in dairy cattle. Journal of American Veterinary Medicine Association., v. 8, p. 845, 1986.

GONZALEZ, F. H. D. O perfil metabólico no estudo de doenças da produção em vacas leiteiras. Arquivo da Faculdade de Veterinária UFRGS, Porto Alegre, v. 25, n. 2, 1997.

GREGORY, L.; JUNIOR, E. H. B.; MIRANDOLA, R. M. S.; ARUJO, W. P.; BIRGEL. E. H. Valores de referência da atividade enzimática da aspartato-aminotransferase e da gama-glutamiltransferase em bovinos da raça Jersey. Influência dos fatores etários, sexuais e da infecção pelo vírus da leucose dos bovinos. Arquivo Brasileiro de Medicina Veterinária e Zootecnia, BH, v. 51, n. 6, p. 512-522, 1999.

GRUMMER, R. R. Etiology of lipidi-related metabolic disorders in periparturient dairy cows. Journal of Dairy Science, v. 76, p. 3882, 1993.

GRUMMER, R. R. Impact of changes organic nutrient metabolism on feeding the transition dairy cow. Journal of Animal Science, v. 73, p. 2820-2833, 1995.

HERDT, T. H. Fuel homeostasis in the ruminant. Veterinary Clinics of North America. Food Animal Practice, v. 4, p. 213-232, 1988. 
JOHNSON, M. M.; PETERS, J. P. Technical note: An improved method to quantify nonesterified fatty acids in bovine plasma. Journal of Animal Science, v. 71, p. 753, 1993.

KANEKO, J. J. Clinical Biochemistry of Domestic Animals. 5. ed., New York, Academic Press, 1997.

KUNZ, P. L.;BLUM, J.W.; HART, I. C.; BICKEL, H.; LANDIS, J. Effects of different energy intakes before and after calving on food intake, performance and blood hormones and metabolites in dairy cows. Animal Production, v. 40, p. 219-231, 1985.

LEHNINGER, A. L.; NELSON, D. L.; COX, M. M. Princípios de bioquímica, 2. ed., São Paulo: Sarvier 1995.

MILLER, P. S.; REIS, B. L.; CALVERT, C. C.; DePETERS, E. J.;BALDWIN, R. L. Relationship of early lactation and bovine somatotropin on nutrient uptake by cow mammary glands. Journal of Dairy Science, v. 74, p. 3800-3806, 1991.

NIELEN, M.; AARTS, M. G. A.; JONKERS, A. G. M.; WENSING, T.; SCHUKKEN, Y. H. Evaluation of two cow side tests for the detection of subclinical ketosis in Dairy cows. Cannadian Veterinary Journal, v. 35, p. 229, 1994.

PUTNAM, D. E.; VARGA, G. A.; DANIN, H. M. Metabolic and production responses to dietary protein and exogenous somatotropin in late gestation dairy cows. Journal of Dairy Science, v. 82, p. 982-995, 1999. RADOSTITS, O. M.; GAY, C. C.; BLOOD, D. C.; HINCHCLIFF, K. W. Veterinary Medicine, 9. ed., W. B. Saunders Company, 2000.

ROBERTS, C. J.; REID, I. M. Fat cow syndrome and subclinical fatty liver. In: HOWARD, J. L. (ED) Current Veterinary Therapy, Food Animal Practice, 2. ed., Philadelphia, W. B. Saunders, 1986. p. 324-326.

RUKKWAMSUK, T.; KROIP, T. A. M.; MEIJER, G. A. L.; WENSING, T. Hepatic Fatty Acid Composition in Periparturient Dairy Cows with Fatty Liver Induced by Intake of a High Energy Diet in the Dry Period. Journal of Dairy Science, v. 82, p. 280-287, 1999.
SANTOS, J. E. P. Effect of degree of fatness prepartum on lactational performance and folicular development of early lactating dairy cows. 1996. $107 \mathrm{f}$. Tese (Mestrado)-University of Arizona, Arizona.

STAFSOFT, Inc., STATISTICA for windows [Computer program manual]. Tulsa, OK: Stafsoft, Inc., 2325 East 13 Street, Tulsa, OK, 74104, (918)583-4149, fax: (918) 583-4376, 1995.

STOBER, M.; GRUNDER, H. D.; DIRKSEN, G. Exame clínico dos bovinos. 3. ed. Rio de Janeiro: Guanabara Koogan, 1993.

TIETZ, N. W. Fundamental of Clinical Chemistry, 2. ed., Philadelphia, Saunders, 1982.

TVEIT, B.; LINGAAS, F.; SVENDSEN, M.; SJAASTA, O. V. Etiology of acetonemia in Norwegian cattle. 1. Effect of Ketogenic silage, season, energy level and genetic factor. Journal Dairy Science, v. 75, p. 24212432, 1992.

VAN SOEST, P. J.; ROBERTSON, J. B.; LEWIS, B. A. Methods for dietary fiber, neutral detergent fiber and nonstarch polysaccharides in relation to animal nutrition. Journal of Dairy Science, v. 74, n. 12, p. 3583 3597,1991

VASQUE-ANON, M.; BERTICS, S.; LUCK, M.; GRUMMER, R. R. Peripartum liver triglyceride and plasma metabolites in dairy cows. Journal Dairy Science, v. 77, p. 1521, 1994.

WHITAKER, D. A. In: ANDREWS, A. H. The health of dairy cattle. Blackwell Science, 2000. p. 89-107.

WILDMAN, E. E.; LONES, G. M.; WAGNER, P. E.;BOMAN, R. L.; TROUT Jr., H. F.; LESCH, T. N. A dairy body condition scoring system and its relationship to selected production characteristics. Journal of Dairy Science, v. 77, p. 1556, 1982.

WILLIANSON, D. H.; MELANBY, J.; KREBS, H. A. Enzymatic determination of $\mathrm{D}(-)$ b-hydroxybutyric acid and acetoacetic acid in blood. Biochimestry Journal, v. 82, p. 90-96, 1962. 